\title{
Ute Gerhard
}

\section{National oder International}

\author{
Die internationalen Beziehungen \\ der deutschen bürgerlichen Frauenbewegung
}

Üblicherweise werden die internationalen Beziehungen und Kooperationen der Frauenbewegung als Beiwerk betrachtet, als eine gewiß interessante, aber gleichwohl zusätzliche oder ergänzende Fragestellung. Einer der Gründe, warum die internationale Geschichte der Frauenbewegungen zumindest aus der deutschen Perspektive bisher nicht untersucht worden ist, warum die internationalen Verbindungen bisher nicht im Zentrum des historischen und politischen Interesses standen, mag darin liegen, da $B$ ihre Bedeutung für die Mobilisierung der Frauenbewegung kaum beachtet wurde. Hinzu kommt, daß speziell im deutschen Kontext Fragen des Nationalismus und Internationalismus eine komplizierte Vorgeschichte haben, aus der auch die Frauengeschichtsforschung nicht heraustreten oder sich gar mit dem Rekurs auf einen spezifisch weiblichen Pazifismus davonstehlen kann. Schließlich gibt es in Anbetracht zweier verheerender und verlorener Weltkriege besonders nach 1945 einen Bedeutungsverlust, einen Niedergang der deutschen Frauenbewegung selbst und ihrer internationalen Reputation. Gerade für die Geschichtsforschung erweist sich der durch Kriegszerstörung unersetzliche Verlust wichtiger Dokumente, ganzer Nachlässe und Archive, aber auch die Zerstreutheit und bisher nicht systematisierte Aufarbeitung der erhaltenen Materialien als eine zusätzliche Schwierigkeit für die Bearbeitung dieses Themas. So bleibt, weil das gerettete Material (Korrespondenzen, Broschüren und Verlautbarungen der internationalen Frauenorganisationen usw.) in alle Kontinente verstreut ist, zur Rekonstruktion dieser Geschichte oft nichts anderes übrig, als den Spuren einiger Emigrantinnen zu folgen oder in amerikanischen Archiven von der frühen Aufmerksamkeit für diesen Teil einer internationalen Frauengeschichte zu profitieren. ' Dieser Tatbestand erklärt aber auch, warum es bisher eigentlich nur angelsächsische Monographien bzw. Selbstdarstellungen zum Thema gibt, in denen nur wenige Hinweise auf deutsche Beteiligungen zu finden sind. ${ }^{2}$ Die einzige Ausnahme und überaus schätzenswerte Vorarbeit ist die leider nicht gedruckte Dissertation von Irmgard Remme aus dem Jahr 1955, die aus der Perspektive der bürgerlichen Frauenbewegung einen sehr gründlichen und informierenden Überblick über die internationalen Beziehungen der deutschen Frauenbewegung zwischen 1888 und 1933 gibt. $^{3}$ Verfügbar sind darüber hinaus einige neuere Editionen mit ausgewählten Quellen, verhältnismäßig wenige Kongreßma- 
terialien $^{5}$ sowie Broschüren, Zeitschriften und nicht erschlossene Korrespondenzen. $^{6}$

Schon ein erster Blick in die bisher aus der deutschen Perspektive nicht aufgearbeitete Geschichte der internationalen Beziehungen und Organisationen der alten Frauenbewegung zeigt, wie vielfältig, intensiv und spannend der Austausch und die Kontakte insbesondere in der Zeit um die Jahrhundertwende bis zum Ersten Weltkrieg waren. Der Aufschwung der nationalen Frauenbewegungen in verschiedenen Ländem am Ende des 19. Jahrhunderts verdankt sich zu einem nicht unwesentlichen Teil dem Wissen um gleiche Problemlagen und dem AnstoB durch übernationale Zusammenschlüsse, also einer »Weltbewegung « der Frauen, um gemeinsam der politischen Unmündigkeit entgegenzutreten und ihren besonderen Anliegen, sei es dem Stimmrecht, der Abschaffung staatlich geregelter Prostitution oder der Verbesserung der sozialen Situation der Frau mehr Schubkraft zu verleihen.

Wir Frauen aller Nationen schließen uns in der Überzeugung, daß das Wohl der Menschheit nur durch eine größere Einheitlichkeit in Gedanken, Gefühlen und Bestrebungen gefördert werden kann und daß eine organisierte Frauenbewegung dem Wohl der Familie und des Staates am besten dienen wird, hiermit zu einem Bunde zusammen...?

heißt es sehr allgemein, aber emphatisch in der Präambel der Satzung des 1888 gegründeten Internationalen Frauenbundes (International Council of Women, ICW). Tatsächlich hatten die Amerikanerinnen von Anfang an die Initiative und damit auch die Führung in dieser internationalen Ausrichtung und Organisation übernommen - und wie in einem 1954 verfaßten Überblick über die »Highlights of the I.C.W. « betont wird - kümmerten sie sich sogar zuerst um die internationale Kooperation, "placing humanity above nationalities ${ }^{8}$, bevor es ihnen gelang, einen nationalen Zusammenschluß der unterschiedlichen, aber auch rivalisierenden amerikanischen Frauenrechtsorganisationen zu erreichen. ${ }^{9}$

Offensichtlich geben die offiziellen Verlautbarungen, Kongreßberichte und Selbstdarstellungen der verschiedenen internationalen Organisationen nur ein sehr einseitiges, unvollständiges und - wegen einer uns heute fremden Rhetorik - allzu abstraktes Bild. Nach allen historischen Erfahrungen, dem Scheitern der besonderen Friedens- und Verständigungsbemühungen in und nach den beiden Weltkriegen gerade auch der Frauen, ergreift uns heute eher eine tiefliegende Skepsis gegenüber einer besonderen internationalen Mission der Frauenbewegungen und dem hoffnungsvollen Überschwang ihrer Vertreterinnen. Gleichzeitig genießen sie unsere politische Sympathie im Gegensatz zu den engstirnigen Verteidigerinnen nationaler oder gar nationalistischer Interessen. Jedenfalls sind die Bewertungen und Beurteilungen nicht unabhängig von den politischen Kontexten und Standorten zu fällen, bleibt dieser Teil der Frauenbewegungsgeschichte ganz besonders eng mit der politischen Geschichte des jeweiligen Landes und dem Frieden oder Unfrieden in der Welt verknüpft. Zugleich ist dieser Aspekt und Beweis frauenpolitischen Engagements bisher weder von den angesprochenen Politikern ${ }^{10}$ noch im nachhinein von der sog. allgemeinen Geschichtswissenschaft berücksichtigt oder beachtet worden. 
Es interessierte mich, welche Bedeutung die internationalen Organisationen und Kooperationen für die Politik der deutschen Frauenbewegung hatten, und wie ihre Rolle von auBen, im erweiterten Horizont der internationalen Beziehungen $\mathrm{zu}$ beurteilen ist. Im folgenden beschränke ich mich auf die bürgerliche Frauenbewegung und versuche anhand bisher kaum zugänglicher Quellen, die Innenansicht und das Selbstverständnis der beteiligten Frauen zu thematisieren. In den Korrespondenzen der Vertreterinnen der Frauenbewegung mit ihren Partnerinnen auf internationaler Ebene erschlieBt sich eine neue Perspektive auf die politische Praxis und die unterschiedlichen nationalen Orientierungen der Beteiligten, erweist sich erneut, wie sehr erst das Netzwerk von persönlichen und zugleich politischen Beziehungen unterhalb der organisatorischen Ebene der Bewegung Wirksamkeit und Anschaulichkeit verleiht. ${ }^{91}$

Die von mir durchgesehene Korrespondenz ${ }^{12}$ - ein Bruchteil des Möglichen, der sich wegen der verfügbaren Quellen eher zufällig auf bestimmte Beziehungen der bürgerlichen Frauenbewegung und vorrangig auf die Organisation des ICW beschränkt - zeugt von der Vielfalt frauenbewegter Kooperation, von der Erfahrung nationale Grenzen überwindender Gemeinsamkeiten und einem sich über diese Internationalität konstituierenden feministischen SelbstbewuBtsein. Der Umgangsstil wirkt zum größten Teil sehr modern, er ist weltläufig und unkonventionell - die Schreiberinnen benutzen jeweils ihre Muttersprache (also Deutsch, Englisch oder Französisch) und dürfen die Kenntnis bei ihren Adressatinnen voraussetzen, d.h. sie schreiben jeweils in ihrer Sprache zuriick. Dies gilt jedoch uneingeschränkt nur bis zum Ersten Weltkrieg und belegt zugleich, welche privilegierenden gesellschaftlichen Voraussetzungen, Bildung, Sprachenkenntnisse und Geldmittel zur Pflege dieser Art Kontakte notwendig waren. Darüber hinaus dokumentiert das historische Material auch die typischen Mißverständnisse, nationalen Empfindlichkeiten und Fremdheiten und spiegelt so im historischen Verlauf die politischen Entwicklungen und internationalen Spannungen und Auseinandersetzungen auf eine sehr persönliche Weise wider.

In Anbetracht der Forschungs- und Materiallücken kann eine erste Annäherung nur sehr provisorisch und unvollständig sein. Im folgenden können daher die Entwicklung und die Brüche in den internationalen Beziehungen nur in groben Ausschnitten skizziert werden. Ich möchte die Fülle des Stoffes um die Bruchstellen der deutschen Geschichte organisieren und in vier Schritte gliedern: Die Zeit vor dem Ersten Weltkrieg, diesen Krieg und seine Folgen, die Zeit des Nationalsozialismus und die Nachkriegszeit ab 1945.

\section{Vor 1914: Aufbruch auch zu internationalen Ufern}

Die Kontakte und neuen Bekanntschaften mit der internationalen Szene des Feminismus waren für den Aufschwung der deutschen Frauenbewegung am Ende des 19. Jahrhunderts nicht nur eine wichtige Anregung und Stütze, sie waren für ihre Organisationsweise geradezu konstitutiv. Nach dem Besuch der 2. Generalversammlung des International Council of Women (ICW) im Zusammenhang mit der Weltausstellung 1893 in Chicago waren die deutschen Teilnehmerinnen (Anna 
Simson, Hanna Bieber-Böhm, Auguste Förster und Dr. Käthe Schirmacher) überhaupt erst auf die Idee gekommen, die seit dem Ende der Bismarck-Ära neuen Initiativen und Frauenvereine zum Dachverband des Bundes Deutscher Frauenvereine (BDF, begründet 1894) zu vereinigen. Seit 1897 war der BDF auf diese Weise auch Mitglied im International Council of Women.

Anläßlich der Gründung des ICW 1888 hatte der Allgemeine Deutsche Frauenverein noch abwehrend und um Verständnis bittend auf die Einladung zur Teilnahme mit dem Hinweis auf die politischen Schwierigkeiten geantwortet: »... in Germany we have to work with great tact and by conservative methods ... The difference between our position and that of our American sisters is largely due to the fact that you live in a republic, we in a monarchy ... « ${ }^{13}$ Tatsächlich war das politische Klima im Wilhelminischen Kaiserreich, das in Konkurrenz zu Frankreich und England zur imperialen Weltmacht aufsteigen wollte, gegenüber allen Bestrebungen um Friedenssicherung und internationale Verständigung keineswegs günstig, im Gegenteil: »International« wurde zugleich als »antinational « denunziert. Von dieser Tendenz zeugt ein Kommentar von Helene Lange, die im Oktober 1900 in ihrer Zeitschrift »Die Frau« schrieb:

Und so löst sich die Frage »National oder International « für die Frauenbewegung dahin: Unzweifelhaft vermittelt die internationale Bewegung den deutschen Frauen wertvolle Einblicke und neue Gesichtspunkte; sie zeigt ihnen zum Teil in die Praxis des täglichen Lebens überführt, was bei uns noch graue Theorie ist. Ohne Gefahr ist sie aber doch nur für die, die fest auf dem Boden des eigenen Volkslebens stehen und die Bedingungen seiner Kultur in Vergangenheit und Gegenwart genügend beherrschen, um kritisch zu sichten, umbilden und anpassen zu können. Bäume brauchen eigenen Boden zum Wurzeln; auf fremden Organismen führen nur Schmarotzer ihr kurzlebiges Dasein. ${ }^{14}$

In dieser Gegenüberstellung und Entgegensetzung von »national« und »international « deutet sich auch in der Frauenbewegung schon sehr früh eine grundlegende Differenz und spezifisch deutsche Entwicklung an, die - um Mißverständnisse zu vermeiden - kurz zu klären ist. Denn die Bezugnahme auf die Nation, die Kennzeichnung nationaler Interessenorganisation ist nicht notwendig mit nationalistischer Politik gleichzusetzen. Kennzeichnete der Begriff Nation seit der Französischen Revolution in der politischen Sprache eine politische Willensgemeinschaft, die sich als allgemeinster Stand oder Staatsvolk gegen den ständischen Privilegienstaat richtete, so waren auch die nationalen Befreiungsbewegungen im 19. Jahrhundert, z.B. in Deutschland die Bestrebungen nach nationaler Einheit um die 1848er Revolution, demokratische Bewegungen, die die Nationenbildung mit dem Prinzip der Volkssouveränität und einer freiheitlichen Verfassung verbanden. Das Scheitern der ersten »National «-Versammlung der Deutschen hatte zur Folge, $\mathrm{da} B$ Deutschland ein unvollendeter Nationalstaat blieb, eine »verspätete Nation ${ }^{15}{ }^{15}$ die im Vergleich zu den westlichen Industrienationen einen "Sonderweg ${ }^{16}{ }^{16}$ beschritt. Als Gründe für diese Negativzuschreibungen werden genannt: seine undemokratische Verfassung als Bund deutscher Fürsten, die fehlende Gleichberechtigung und politische Beteiligung seiner Bürger und Bürgerinnen (z.B. das Dreiklassenwahlrecht in Preußen, kein Wahlrecht für Frauen), die durch 
Klassengegensätze gespaltene Nation. Spätestens seit der Reichsgründung 1871 aber machte sich gegen die Einlösung der nationaldemokratischen Zielsetzungen ein »organisierter Nationalismus « von rechts breit, der mit dem Begriff »deutsch « $=$ " volkdeutsch «, »deutsch-national $\ll$ nicht mehr nationale Integration, sondern ethnische Ausgrenzung verband und sich nicht nur durch einen neu begründeten Antisemitismus, sondern einen ebenso militanten Antifeminismus hervortat. ${ }^{17}$

Trotzdem ist auch nach 1890 auf den Aufschwung des Vereinswesens und auf außerparlamentarische Bewegungen hinzuweisen, die für die demokratische Reform der deutschen Monarchie, für die Emanzipation und Gleichberechtigung aller Mitglieder, für ein gleiches Staatsbürgertum eintraten. Sie kamen aus dem Lager der Linksliberalen, der Gewerkschaften, umfaßten auch Teile der sich zur Reformpartei entwickelnden SPD, aber nicht zuletzt die seit 1890 erstarkende Frauenbewegung. Sie hatte schließlich schon 1865 , vor der Reichsgründung, mit der Gründung des Allgemeinen Deutschen Frauenvereins in Leipzig eine nationale Organisationsform gefunden, ohne doch nationalistisch zu sein. Der Bund Deutscher Frauenvereine (1894), ja, auch der radikalere Deutsche Verein für Frauenstimmrecht (1902) verstanden sich als Vertreter eines politischen Liberalismus, dessen Ideale erst noch zu verwirklichen wären. ${ }^{18}$ Sie kämpften um die Erweiterung der Rechte von Frauen in diesem Staat, um Zulassungen und staatliche Berechtigungen, damit aber auch um grundlegende Veränderungen dieses Staatswesens. In der Frauenbewegung strittig war, wie diese Gesellschaftsreform zu verwirklichen sei, inwieweit die universalen Prinzipien der Freiheit und Gleichheit und allgemeiner Menschenrechte auch in diesem Kaiserreich Gültigkeit beanspruchen konnten. ${ }^{19}$ Die Antwort auf diese Fragen war ausschlaggebend auch für die Bereitschaft zu internationaler Kooperation und bestimmte die Differenzen zwischen den Gemäßigten und Radikalen, aber auch die Abgrenzung zu den mehr international orientierten Sozialistinnen.

In Helene Langes Kommentar ist die Entgegensetzung von Nationalismus und Internationalismus in vielen assoziativen und polemischen Formulierungen vorgezeichnet. Nicht nur die Beschwörung einer Gefahr, die vom Internationalismus ausgehe, das Bild vom »eigenen Boden« und den »Schmarotzern« sind Sprachregelungen, die bestimmte Gruppen auch aus der sog. Volksgemeinschaft ausgrenzen und als »Andersartige « identifizieren. Kein Klischee ist im gleichen Kommentar zu billig, um das "Nationalgefühl « auch der Frauen zu charakterisieren: »der Erdgeruch der heimischen Scholle, das Rauschen des deutschen Waldes, das Bewußtsein einer eigenen Stammesindividualität ... « usf. ${ }^{20}$

Dagegen beklagte Marie Stritt, die sich zwischen 1899 und 1910 als Vorsitzende des BDF persönlich sehr für den internationalen Austausch engagierte und die Beziehungen mit einer ausgedehnten Korrespondenz pflegte, das nur vereinzelte Eintreten der Frauen für internationale, insbesondere pazifistische Ziele. Sie suchte nach den Gründen für diesen bornierten und reaktionären Nationalismus gerade in Deutschland:

Vielleicht liegt es daran, daß seit dem Kriege 1870/71 im deutschen Volksempfinden - also auch im Empfinden der Frauen - alle Vorstellungen von nationaler Größe und Wohlfahrt an den Militarismus geknüpft sind, und daß man demzufolge 
den Gedanken des Internationalismus, auf dem alle Friedensbestrebungen basieren, selbst in aufgeklärten Kreisen als Utopie betrachtet ... ${ }^{21}$

Ohne Zweifel hat der programmatische Internationalismus der Arbeiterbewegung, die Sozialistische Internationale, und die klassenkämpferisch auch in der Frauenbewegung betonte »reinliche Scheidung " 22 im bürgerlichen Lager zusätzlich zur Skepsis und Denunziation internationaler Bestrebungen als "antinational « oder »vaterlandslos « beigetragen. Doch auch die Proletarierinnen haben schließlich auf der 1. Internationalen Frauenkonferenz in Stuttgart 1907 »das volle Bürgerrecht, die volle politische Gleichberechtigung des weiblichen Geschlechts « auf nationaler Ebene gefordert, wobei sie im Unterschied zu den Frauen des ICW Frauenrechte, insbesondere das Frauenwahlrecht ohne Rücksicht auf die politischen Verhältnisse in den einzelnen Gesellschaften beanspruchten. ${ }^{23}$

Wie ernsthaft sich dennoch seit der Jahrhundertwende führende Feministinnen an dem internationalen Gedankenaustausch und der Übung in Frauensolidarität $\mathrm{zu}$ beteiligen suchten, ist an ihrer regen Korrespondenz und der eifrigen Teilnah$\mathrm{me}$, ja, bravourösen Organisation verschiedener internationaler Kongresse - 1896, 1904, 1912, alle in Berlin - abzulesen. Im Gegensatz zu den Briefen der Amerikanerinnen wirken die ersten Antworten der deutschen Korrespondentinnen reservierter, steifer, ziemlich nüchtern. Da werden die Formalien streng beachtet, die Auswahl der Delegierten und die Redezeiten ausgehandelt, Anträge und Resolutionen sorgfältig vorbereitet. Bemerkenswert ist, wie empfindlich die stellvertretende Vorsitzende des BDF, Anna Simson, 1899 im Vorfeld des Londoner Kongresses auf das Ersuchen der englischen und amerikanischen Schwestern reagierte, Lady Aberdeen, eine Aristokratin, erneut für die Präsidentschaft im ICW vorzuschlagen. Die Antwort ist nur aus dem Selbstverständnis der deutschen Frauenbewegung als »bürgerliche«, emanzipatorische, den Prinzipien der Aufklärung und Demokratie verpflichtete zu interpretieren, die gerade weil sie noch in einer Monarchie mit feudalen Privilegien und Strukturen zu kämpfen hatte, in der Frage der Präsidentschaft so reagierte:

Die deutsche Frauenbewegung ist eben eine durch und durch bürgerliche und steht als Frauenbewegung allen aristokratischen, konservativen und kirchlichen Wohltätigkeitsbestrebungen (ablehnend) gegenüber ... (und bezogen auf eine andere adlige Kandidatin, die Finnin Alexandra Baronin Gripenberg) ... die ich persönlich sehr hoch schätze, so möchte ich noch hinzufügen, daß dieser Adel, als Adel, in Deutschland absolut nichts bedeutet. ${ }^{24}$

Auch in diesem Brief kommt zur Sprache, daß »internationales « Engagement gerade in den konservativen Kreisen immer zugleich als »antinational « gedeutet wird, was für den BDF ein Grund mehr war, sich nicht durch »diesen « Adel vertreten zu lassen. Ja, die Hoffnung der deutschen Bürgerlichen auf Rückenstärkung in demokratischer Absicht ging offenbar noch weiter. In einer ausführlichen Notiz der ehemaligen Vorsitzenden des BDF an die Nachfolgerin Marie Stritt spricht Auguste Schmidt ihre Enttäuschung über die bisherige Zusammenarbeit im ICW sehr nüchtern aus. Die erneute Präsidentschaft einer Engländerin wie 
Lady Aberdeen würde ihrer Meinung nach das für den Weltbund angemessene „Prinzip der Wechselherrschaft zwischen den verschiedenen Nationen « verletzen und die Vorherrschaft Englands stärken. Da Deutschland sich bereit erklärt hatte, die nächste Generalversammlung in Berlin im Jahr 1904 zu veranstalten, hätte es nahegelegen, Deutschland für die nächsten fünf Jahre die Präsidentschaft anzutragen. Doch Auguste Schmidt war realistisch genug anzunehmen, „daB bei der jetzigen Weltlage keine der stimmberechtigten Damen ihre Stimme Deutschland geben wird «. ${ }^{25}$ Mit den Stimmen der Deutschen wurde darum 1899 in London die Amerikanerin May Wright Sewall zur Präsidentin für fünf Jahre gewählt, um dann dieses Amt von 1904 bis 1936 doch einer Lady, der Marquise Isabel von Aberdeen und Temair, zu überlassen, die damit - nur mit einer kurzen Unterbrechung zwischen 1920 und 1922 - über alle Zeiten und Dramen hinweg bis 1936 den ICW anführte.

Neben der sehr allgemeinen und unverfänglichen »Goldenen Regel $\ll$, die in allen Briefen der Präsidentin als Motto zu lesen war: "Do unto others as ye would that others should do unto you $«{ }^{26}$ sah die Verfassung des ICW vor:

1. Der Internationale Frauenbund wurde nicht im Interesse einer bestimmten Propaganda gegründet und schließt aus seinem Programm alle politischen und religiösen Streitfragen aus, die die Beziehungen zweier oder mehrerer Länder untereinander berühren.

2. Der Internationale Frauenbund hat keinerlei Machtbefugnisse gegenüber seinen Mitgliedern. Er kann nur Anregungen geben und freundschaftliche Beziehungen pflegen ... ${ }^{27}$

Diese Verpflichtung des ICW zu Nichteinmischung und absoluter Neutralität in allen politischen Fragen aber bedeutete Handlungsfähigkeit nur auf dem kleinsten gemeinsamen Nenner. Doch weil mit dem Eintreten für mehr Recht, insbesondere das Wahlrecht, politische Forderungen auf der Tagesordnung standen, drängten die radikalen Demokratinnen auch auf internationaler Ebene zunehmend auf eine entschiedenere Politik, um vorbehaltlos für die Rechte der Frauen, insbesondere für das allgemeine Wahlrecht einzutreten. Deshalb wurde 1904 - im engen und nicht konfliktfreien Zusammenhang mit dem Weltkongre B des ICW in Berlin mit der 84jährigen Susan B. Anthony in der Führung und der sehr aktiven Beteiligung der deutschen Stimmrechtlerinnen die International Woman Suffrage Alliance, später International Alliance of Women for Suffrage and Equal Citizenship (IAW) mit der Präsidentin Carry Chapman Catt ins Leben gerufen. ${ }^{28}$ Auch der internationalen Stimmrechtsbewegung gehörten 1909 bereits ebenso viele nationale Verbände wie dem ICW an, nämlich Frauenorganisationen aus 21 Staaten. ${ }^{29}$

Deutlich wird in diesem Aufbau internationaler Beziehungen bis 1914 ein Lerneffekt. Die Zusammenarbeit und das persönliche Kennenlernen werden auf beiden Seiten als Gewinn verbucht. Die »Universität« der Frauenbewegung, die "Einheitlichkeit der modernen Frauenbestrebungen« wird zur "Gewißheit«, die das eigene Tun bestärkt. ${ }^{30}$ Der Glanz internationaler Konferenzen hat eine mobilisierende Wirkung und wird gezielt eingesetzt, "to teach women of different 
countries how to concentrate their efforts on a common object«. ${ }^{31}$ Der zum Teil überschwengliche Ton der Amerikanerinnen wird auch von den Deutschen zunehmend herzlicher, nicht mehr so förmlich beantwortet. "Although nearly strangers, we are friends", schreibt May W. Sewall an Marie Stritt. ${ }^{32}$ Diese antwortet ungezwungen und warmherzig:

Zunächst möchte ich Ihnen herzlich für Ihre freundlichen Zeilen (danken), die dem Gefühl der Zusammengehörigkeit und Solidarität, das uns alle erfüllt, in so liebenswürdiger Weise Ausdruck geben. Ihre freundschaftliche Gesinnung findet bei mir einen starken Widerhall, und es ist mir eine große Freude, mit Ihnen zusammenarbeiten zu dürfen. ${ }^{33}$

Interessant sind manche Nebenbemerkungen, Urteile über die Besonderheiten und Einschätzungen der Frauenbewegung, die sich in ihrem Briefwechsel finden, so etwa, wenn Marie Stritt differenzierend auf eine der vielen Umfragen für den internationalen Geschäftsbericht antwortet und die deutschen Verhältnisse beschreibt:

Von den vielen Tausenden, die heute schon an den verschiedenen Bestrebungen der Frauenbewegung beteiligt sind, dürfte allerdings nur ein verhältnismäßig kleiner Teil zu den eigentlichen Feministen zu rechnen sein, $d . h . z u$ denjenigen, die alle Konsequenzen aus dem Prinzip der Gleichberechtigung der Geschlechter ziehen und sich bewußt sind, daß dies Prinzip eine völlig veränderte Gesellschaftsordnung involviert. ${ }^{34}$

Geradezu missionarisch mutet der Eifer an, mit dem die Amerikanerinnen immer wieder ihre deutschen Kolleginnen über Internationalismus belehren, z. B. May W. Sewall in einem Brief an Anna Simson aus dem Jahr 1899: "I certainly do believe in International amity ... We must learn to feel that our humanity is above our nationality; we must not love the latter less, but we must learn to love the former more ${ }^{35}{ }^{35}$ Mit dem Ausbruch des Ersten Weltkrieges wurde der politische Gehalt solcher Frauenfreundschaften und Freundinnenkultur ${ }^{36}$ auf die Bewährungsprobe gestellt. Nun gewannen die die Überhand, für die das "Zugehörigkeitsgefühl der Frau zur eigenen Nation« ohnehin im Vordergrund stand.

Wenn es zum Wesen der deutschen Frauenbewegung gehört, daß sie sehr gründlich arbeitet ... so liegt es schon in dieser unserer Arbeitsweise begründet, daß die unvermeidliche Flachheit solcher internationalen Arbeit uns bedrückt und widerstrebt. ${ }^{37}$

Mit scharfer Zunge hatte Helene Lange ja schon früher »die Oberflächlichkeit« und das »Phrasentum « der internationalen Kongresse gerügt. ${ }^{38}$ Noch vor Ausbruch des Weltkrieges mahnte auch Gertrud Bäumer ihre Gefolgschaft an die nationale Verpflichtung der deutschen Frauenbewegung in der aus- und abgrenzenden, für den rechten Nationalismus typischen Diktion: 
Eine Bewegung, die nicht nur große schematische wirtschaftliche Forderungen, sondern ein Kulturideal aufstellt, ist schon dadurch in ihrer Verkörperung an den nationalen Charakter gebunden. Soweit die Frauenbewegung sich in leibhafter Gestalt, sich in weiblichen Typen neuer Art und Kraft ausdrücken will ..., ist sie notwendig national, baut sie sich auf vom Blut und Leben des eigenen Volkes. ${ }^{39}$

\section{Bewährungsprobe und Scheitern der Frauensolidarität}

Nicht nur die Politik der internationalen Frauenorganisationen hat 1914 versagt und mögliche Chancen verspielt. Die vielfältigen Ursachen dieses katastrophalen Scheiterns einer Machtpolitik sind untersucht und bekannt. Belegt ist die patriotische Begeisterung, die »seelische Mobilmachung « gerade auch von Frauen, die diese Zeit als "große Zeit « erlebten, in der es darum ging, alle »Sonderinteressen « zurückzustellen und ihre Leistungsfähigkeit und nationale Zuverlässigkeit zu beweisen. Praktisch wird dieses Aufgehen im »Volkskörper« in der Arbeit im Nationalen Frauendienst, unentbehrlich für die Kriegsziele blieb das Durchhaltevermögen der Frauen an der »Heimatfront $~^{40}$.

Grundsätzlich zu erörtern ist natürlich die Frage, welche Macht- oder gar Rechtsmittel Frauen als nicht Stimmberechtigte und von politischer Partizipation Ausgeschlossene überhaupt hatten, um sich gegen den Krieg zu wehren, seiner Ausdehnung und Verlängerung entgegenzutreten. Boten nicht wenigstens die internationalen Organisationen ein Forum oder Medien für Widerstand und Gegenöffentlichkeit? - Zu berücksichtigen ist, daß der Ausbruch des Krieges gerade den internationalen Kontakt durch Zensur und Propaganda, die Schließung der Grenzen, zunehmende Verfolgung und Schikanen gegenüber allen Friedenssuchenden und Pazifisten verhindert bzw. unendlich erschwert hat. Hatte noch im Sommer 1914 kurz vor Kriegsausbruch die Generalversammlung des ICW in Rom die Frauen aus 21 Mitgliedstaaten zu Festbanketten und der Demonstration von Frauensolidarität vereinigt, ohne zu ahnen oder zu diskutieren, $\gg \mathrm{da} B$ die Weltkatastrophe so nahe bevorstehen könnte «, ${ }^{41}$ so sah sich Marie Stritt als Vorsitzende des Deutschen Verbandes für Frauenstimmrecht schon im September 1914 gezwungen, die für 1915 in Berlin zum Weltkongreß eingeladenen Gäste wieder auszuladen. Auch die Präsidentin des Weltbundes für Frauenstimmrecht Carry Chapman Catt glaubte nach einer Umfrage unter den Mitgliedsverbänden, einen internationalen Kongre $B$ nicht unter Kriegsbedingungen veranstalten zu können. Noch im Juli 1914 hatte sie in einem Manifest an das Britische Außenministerium und verschiedene Botschafter in London appelliert: "We, the women of the world ... appeal to you to leave untried no method of conciliation or arbitration which may help to avert deluging half the civilised world in blood. ${ }^{42}$ Immerhin versuchte die Geschäftsstelle der IAW, auch während des Weltkrieges die Kommunikation aufrechtzuerhalten. »It is for us to prove that which binds us is stronger than that which separates «, schrieb die englische Vizepräsidentin Millicent G. Fawcett in der auch im Krieg weltweit verbreiteten Verbandszeitschrift »Jus Suffragii «. ${ }^{43}$ Doch erst der besonderen Initiative und Hartnäckigkeit von Aletta Jacobs und Anita Augspurg war es zu verdanken, daB im April 1915 in Den Haag 
ohne die offizielle Beteiligung der IAW und seiner Präsidentin Chapman Catt, doch getragen von vielen Stimmrechtlerinnen, eine außerordentliche Konferenz für den Frieden unter Beteiligung von 1200 Delegierten zustande kam. ${ }^{44}$

Es entstand hieraus eine dritte, radikalere Frauengemeinschaft: Die Internationale Frauenliga für Frieden und Freiheit (IFFF, seit 1919), bei der auf amerikanischer Seite die Women's Peace Party unter der Führung von Jane Addams beteiligt war. Kennzeichnend für den Kongreß war die Ernsthaftigkeit und Grundsätzlichkeit der hier verabschiedeten Resolutionen, in denen die Forderung nach dem Stimmrecht und der Beteiligung von Frauen an der Politik mit dem Eintreten für eine übernational zu organisierende Schiedsgerichtsbarkeit zur Beilegung internationaler Streitigkeiten verknüpft wurde. Ungewöhnlich war auch der Versuch, durch die Entsendung einer Frauendelegation die Regierungen von 14 Nationen unmittelbar zu überzeugen und für die Beendigung des Krieges zu gewinnen. ${ }^{45}$

Die internationalen Korrespondenzen in dieser Zeit sind zwangsläufig rar. Eine Ausnahme ist ein kurzer hellsichtiger Brief Anna Edingers an May R. Sewall schon aus dem Jahr 1912, in dem sie die Lage sehr realistisch einschätzt:

... Bei uns in Deutschland ist die Propaganda für den Frieden recht schwierig; leider ist in manchen Kreisen der Begriff der Vaterlandsliebe noch zu eng mit dem der Kampfbereitschaft verbunden, so daß denjenigen, die den Krieg verabscheuen, leicht Mangel an Vaterlandsliebe vorgeworfen wird. Es gilt also, in der jungen Generation die Schar derer zu mehren, die auch anders denkenden Kameraden gegenüber an der Überzeugung festhalten, daß der Krieg eine zu überwindende Barbarei ist, die es ernst nehmen mit dem Gebot: Du sollst nicht töten. ${ }^{46}$

Während der internationale Stimmrechtsbund durch seine Publikation »Jus Suffragii« zumindest außerhalb Deutschlands für Zusammenhalt und Informationen sorgte, legt der ICW mit dem Ausbruch des Krieges seine internationalen Beziehungen auf Eis. Lady Aberdeen schrieb im April 1915 an die Vorsitzende des BDF, Gertrud Bäumer:

Ich bin gewiß, daß alle Mitglieder des Internationalen Bundes an dem Kummer teilnehmen, der uns alle betroffen hat, und der uns zwingt, unsere gemeinsame Arbeit und den Verkehr miteinander zu unterbrechen ... Wir alle sind froh darüber, daß die Frauen überall in der Welt dem Ruf der Pflicht und der Opferbereitschaft in so wunderbarer Weise gefolgt sind, und daß sie bewiesen haben, wie sehr sie für jede Art von Hilfs- und Notstandsarbeit ... fähig und bereit waren. ${ }^{47}$

Mit dieser anscheinend wieder selbstverständlichen Übernahme weiblicher Pflichten und Opferbereitschaft aber war der Gegensatz bezeichnet, der sich nun zwischen der Minderheit der Radikalen und Kriegsgegnerinnen und der Masse der Frauen auftat. Die radikalen Pazifistinnen wollten »keine Arbeit für direkte Kriegsziele leisten«, auch nicht Hospitaldienst, "halbtot geschundene Menschen wieder lebendig und gesund machen, um sie abermals den gleichen oder schlimmeren Qualen auszusetzen .... ${ }^{48}$ Die deutschen Teilnehmerinnen des Haager Friedenskongresses wurden nicht nur von der Staatsgewalt verfolgt und über- 
wacht, ihre Briefe zensiert, auch der BDF-Vorstand kam zu der Überzeugung, daß dieses internationale Engagement mit »väterländischer Gesinnung « unvereinbar wäre. ${ }^{49}$ Ebenso erging es den radikalen Sozialistinnen wie Rosa Luxemburg oder Clara Zetkin, die sich nicht dem "Burgfrieden « zwischen Kaiserreich und Sozialdemokratie anschlossen, sondern - fast zeitgleich mit dem Haager Frauenkongre $B$ - eine Internationale Friedenskonferenz sozialistischer Frauen in Bern organisierten und wegen ihrer Antikriegspropaganda verhaftet und verfolgt wurden.

Mit der Niederlage Deutschlands, der Ausrufung der Republik durch den Rat der Volksbeauftragten und damit dem Zusammenbruch der alten Weltordnung nun sollte man meinen, daß die politischen Vorzeichen verändert und endlich die alten Fronten und Streitfragen obsolet geworden wären. Tatsächlich wurden die Karten neu gemischt, doch auch nach dem Erreichen des Stimmrechts für die Frauen wurden die Stimmrechtlerinnen sowie die linken und radikalen Pazifistinnen keineswegs politisch rehabiliert. So erscheint es zwar widersinnig, ist aber Teil dieser Geschichte, daß in Deutschland eher die konservativen Frauen, die noch bis zuletzt das allgemeine und gleiche Frauenstimmrecht vehement abgelehnt hatten, zu den ersten gewählten Volksvertreterinnen gehörten (z.B. Paula MüllerOtfried), während es keine der radikalen Feministinnen schaffte, über die von Männern dominierten Listen und Parteien in den Reichstag zu kommen. Unter den drei großen internationalen Organisationen, dem ICW, der IAW und der Internationalen Frauenliga waren nach 1918 nicht nur die Zielsetzungen neu zu formulieren, auch personell fanden speziell in der deutschen Szene Umbesetzungen und Verschiebungen statt, die auf den ersten Blick durchaus verwirrend sind.

Mit der Erlangung des Frauenwahlrechts vor allem in den in der Stimmrechtsbewegung führenden Ländern (nach Skandinavien vor allem in den USA, Kanada, England, Niederlande, Dänemark, Deutschland, Österreich, Ungarn etc.) war das Hauptziel des Stimmrechtsbundes erreicht, und es stellte sich die Frage seiner Weiterexistenz. Auf dem 1920 in Genf erstmals nach dem Krieg veranstalteten Kongre $B$ aber kamen die Delegierten überein, da $B$ es noch genug zu tun gab, um das Stimmrecht der Frauen aller Nationen zu sichern, um andererseits all die Reformen durchzusetzen, die notwendig wären, um die tatsächliche Gleichheit im Hinblick auf Freiheit, Status und Chancen zwischen Männern und Frauen zu erreichen. ${ }^{50} \mathrm{Da}$ sich die deutschen Stimmrechtsvereine aber bereits 1918 aufgelöst hatten, übernahm nun der Staatsbürgerinnenverband, der die Nachfolge des Allgemeinen Deutschen Frauenvereins (ADF, gegründet 1865) angetreten hatte, die Ämter und Vertretung der Deutschen in der IAW. So kam es, daß »der älteste und konservativste Verein der deutschen Frauenbewegung international das Erbe ihres jüngsten und radikalsten Zweiges (der Stimmrechtsbewegung) antrat ${ }^{51}{ }^{51}$ und damit Gertrud Bäumer bei der internationalen Vertretung der Stimmrechtlerinnen in die Fußstapfen von Anita Augspurg trat. Mit der Umbenennung der International Women Suffrage Alliance in International Alliance of Women for Suffrage and Equal Citizenship im Jahr 1926 fand zugleich eine Annäherung an die Arbeitsweise und allgemeineren Zielsetzungen des ICW statt, fanden immer häufiger gemeinsame Treffen statt, wurde zur besseren Vertretung im Völkerbund sogar ein Gemeinsamer AusschuB für internationale Frauenorganisationen gegründet. 
Dabei hatte es der BDF nach dem Weltkrieg noch 1920 Alice Salomon, der langjährigen Schriftführerin des ICW, untersagt, an der ersten Generalversammlung des ICW nach dem Krieg in Norwegen teilzunehmen. Und dies, obwohl der ICW als eine der ersten internationalen Organisationen für eine völlig gleichberechtigte Teilnahme Deutschlands eingetreten war. »For our ICW there has been no war ... All of our councils are allied; each equally with all the others «, hatte M. W. Sewall schon 1919 in einem persönlichen Brief an den BDF betont. ${ }^{52}$ In der Ausschaltung der Person A. Salomon durch die BDF-Führung, unter besonderer Beteiligung von Gertrud Bäumer, wurde hier zum erstenmal deren jüdische Abstammung zur Sprache gebracht. ${ }^{53}$ Offiziell aber lautete die Begründung, daB auf der Un-Rechtsbasis des Versailler Vertrages und des Ausschlusses Deutschlands vom Völkerbund »eine freie und unbefangene Zusammenarbeit der Frauen verschiedener Länder « noch nicht möglich sei. ${ }^{54} 1925$ nahm erstmals wieder eine achtköpfige deutsche Delegation an der Generalversammlung des ICW in Washington teil. Immer noch bestimmte die Nichtanerkennung der Kriegsschuld und des Versailler Friedensvertrages die Handlungsweise der deutschen Frauen. Bei der Abstimmung eines Antrages zur Abrüstung und ihrer Kontrolle enthielten sich die deutschen Frauen, weil sie in der Forderung nach Einschränkung der Rüstungen eine Ungerechtigkeit sahen gegenüber den Ländern, die (wie Deutschland) ohnehin zu einer völligen Abrüstung gezwungen waren. »Alle Länder sollten ihrer Meinung nach den gleichen Abrüstungsbedingungen unterliegen und nicht einige nach Belieben den Grad der Sicherheit bestimmen. ${ }^{55}$ Eine große Presse im In- und Ausland fand der erste öffentliche Auftritt einer deutschen Delegation des Staatsbürgerinnenverbandes auf dem Internationalen Kongreß der IAW 1926 in Paris, da Gertrud Bäumer hier, an die Französinnen gewandt, versöhnliche Worte fand, woraufhin die französische Vorsitzende der Allianz Bäumer demonstrativ umarmte.

Die Pazifistinnen, auch die in der Zeit der Weimarer Republik so rührige Internationale Frauenliga für Frieden und Freiheit (IFFF, englisch: WILPF = Women's International League for Peace and Freedom) blieben weltweit eine Minderheit. In Deutschland waren sie zunehmend isoliert, trotz vielfältiger Aktivitäten für eine "Völkerversöhnende Frauenarbeit «, ${ }^{56}$ einer eigenen Presse (z.B. der Zeitschrift »Die Frau im Staat «), sowie bedeutender Kongresse zur Abrüstung und gegen den Gaskrieg und die sog. »modernen Kriegsmethoden « (1929 und 1932). Schon 1924 richteten sie eine »Kommission zur Bekämpfung des Antisemitismus« ein. $\mathrm{Zu}$ ihrer Isolation trug gerade mit dem manifester werdenden Nationalismus ohne Zweifel auch der oberste Grundsatz der IFFF bei, der forderte, dem Internationalismus den Vorrang vor Nationalismus zu geben. ${ }^{57}$ Die unermüdlichen »Women workers «, wie sich die Protagonistinnen im internationalen Jargon nannten, aber scheinen zumindest in den zwanziger Jahren trotz aller Enttäuschungen nicht resigniert zu haben. Aus einem Brief Lida G. Heymanns an Rosika Schwimmer in den USA war u.a. zu erfahren:

Unsere Liga-Arbeit in Deutschland macht trotz schlechter wirtschafticher Verhältnisse, in unserer Kasse herrscht ewig Ebbe, gute Fortschritte. Unsere radikale Art Pazifismus zu vertreten, fordert selbst die Achtung unserer Gegner heraus. ... 
Außer der Arbeit brachte 1927 uns auch viel Schönes, wir reisten 10 Wochen, waren in Egypten, Palästina, Griechenland und Jugoslawien. In Palästina und Jugoslawien hatten wir Gelegenheit für unsere Liga zu arbeiten.$^{58}$

Beeindruckend bleibt der dramatische Appell, den Lida G. Heymann in der Zeitschrift »Die Frau im Staat 1932 an die Welt richtet, die vielfältigen Friedensund Verständigungsbemühungen ihrer Liga resümierend: "S.O.S. Der Zeiger steht auf zwölf, entsagt der Gewalt auf ganzer Linie! Soll Frauenruf abermals ungehört verhallen? $\ll^{59}$

Im Überblick über diesen Zeitabschnitt ist bemerkenswert, daß die deutsche Frauenbewegung auf der internationalen Bühne seit dem Ende des Ersten Weltkrieges ihren mitgestaltenden EinfluB verloren hatte und in den Gremien nicht nur in sehr viel geringerer Zahl als vor dem Krieg vertreten war, sondern sich auch mit unbedeutenden Ämtern zufrieden geben mußte. Eigentlich war es dann auch in beiden internationalen Organisationen, dem ICW und der IAW, eine Handvoll führender Frauen, immer die gleichen Funktionärinnen, die dieses eher lästige Geschäft vor allem in dem Bemühen betrieben, vor der deutschen Öffentlichkeit "auf ihrem nationalen Standpunkt zu beharren $«{ }^{60}$ Diese politische Rückschrittlichkeit und die Nachwuchssorgen der Frauenbewegung zeigten daher zugleich eine Schwäche des Feminismus an, dem es in dieser Zeit offenbar auch über die internationalen Beziehungen und auch in den anderen Ländern nicht mehr gelang, eine einigende oder gemeinsame Plattform zu finden.

\section{1933: Selbstauflösung, Gleichschaltung und internationale Isolierung}

Die nationalsozialistische Machtergreifung bedeutete den einseitigen Abbruch internationaler Beziehungen und das Ende des Feminismus in Deutschland. Der Bund Deutscher Frauenvereine war der Gleichschaltung durch das NS-Regime durch Selbstauflösung zuvorgekommen, damit auch als Vertretung in ICW und IAW ausgeschieden. Auf den internationalen Zusammenkünften waren die deutschen Frauen von nun an grundsätzlich nicht mehr vertreten (nur ausnahmsweise noch als Privatpersonen, z.B. Dorothee von Velsen 1934 auf der Generalversammlung des ICW in Istanbul). ${ }^{61}$ Die NS-Frauenschaft hatte die Dreistigkeit besessen, sich um Aufnahme in den ICW zu bemühen, war aber von Lady Aberdeen mit dem Hinweis auf die Verletzung der Goldenen Regel durch die Nazis abgelehnt worden. ${ }^{62}$ In den Berichten über internationale Kongresse wird die Abwesenheit der Deutschen m.W. in jener Zeit nicht einmal kommentiert, geschweige denn zum Nationalsozialismus Stellung bezogen.

Die führenden Vertreterinnen der Internationalen Frauenliga (IFFF) waren, um der Verfolgung zu entgehen, wie die Sozialistinnen zur Emigration gezwungen, ohnehin dann, wenn sie Jüdinnen waren. Von dem zentralen Büro in Genf aus wo sich die IFFF mit ihrem »Maison Internationale « eine Stätte der Begegnung und der Frauenkultur geschaffen hatte - wurde ein Netz internationaler Solidarität weit und wirkungsvoll um viele Einzelschicksale in der Emigration gespannt. Wie die noch kaum ausgewerteten Dokumente zeigen, spielte inshesondere Gertrud 
Baer während des Zweiten Weltkrieges als Verbindungsperson in den USA eine bewundernswerte Rolle. In ihren "International Circular Letters" gelang es ihr, die IFFF durch gründliche und zuverlässige Informationen nüchtern zusammenzuhalten und im kritischen Umgang mit den nationalen Begrenzungen und Egoismen, insbesondere auch der Amerikanerinnen, für die Emigrantinnen aus Europa viel Gutes zu stiften. ${ }^{63}$ Anrührend und beklemmend sind hingegen die zahlreichen Bittsteller-Briefe z.B. im NachlaB der Rosika Schwimmer von ehemals selbstbewußten und ungewöhnlichen Frauen, die nun als Emigrantinnen - meistens mittellos - zur Einreise ein Affidavit, d.i. die Bürgschaft von zwei Amerikanerinnen, benötigten, ${ }^{64}$ - eine erneute Bewährungsprobe internationaler Schwesternschaft, die bisher viel zu wenig untersucht wurde.

In Genf spielten auch die in die Schweiz emigrierten Feministinnen Anita Augspurg und Lida G. Heymann als Ehrenpräsidentinnen der Frauenliga noch eine gewisse Rolle. So wird z.B. die achtzigjährige Augspurg geschildert als »one of the founders of the WILPF (who) did not miss any opportunity of reminding especially the younger women, that peace without freedom is a shame, and that a just and permanent peace can only be established on a basis of freedom fully acquired and in safe possession ${ }^{65}$. Aus den Briefen an Rosika Schwimmer ist auch zum erstenmal etwas über die Haltung und Überzeugungen von Anita Augspurg und Lida G. Heymann in der Emigration zu erfahren. Die Schreiberin ist jeweils Lida G. Heymann, doch wie selbstverständlich sie auch für A. Augspurg spricht, wird verschiedentlich an der Unterschrift mit zusammengezogenen Vornamen »Anilid « deutlich. Wie auch in den Lebenserinnerungen der beiden Freundinnen zum Ausdruck kommt, ${ }^{66}$ sind sie sich selbst und ihren pazifistischen und radikalfeministischen Überzeugungen treu geblieben. Doch ihr Entsetzen über den Zustand der Welt wirkt in den Briefen noch unmittelbarer und authentischer:

Das Jahr 1938 hat wahrlich schlimm begonnen! Diese Verbrecher: Mussolini, Hitler und Stalin können sich alles erlauben! Das Weltgewissen schläft weiter. Die männlichen Politiker sind mit unbegreiflicher Blindheit geschlagen. Was hier in Europa vorgeht, ist katastrophal. Man staunt ..., daß alles so weitergeht. Augspurg und ich haben unsere Gesinnung, was Pazifismus betriff, nicht geändert. Wir sind heute wie 1915 der Ansicht, daß durch Gewalt weder in der Erziehung noch zwischen Menschen und Völkern Gutes geschaffen werden kann. ${ }^{67}$

Beachtlich ist, daß die Pazifistin im Krieg und zwar im Jahr 1942 ihre Meinung zum absoluten Gewaltverzicht deutlich modifiziert hat. Wiederum ist die Sprache direkt und schonungslos:

Meine Erfahrung hat mich aber gelehrt, daß die Völker - von den Regierungen ganz zu schweigen - noch lange nicht diesen Grad (überlegener Ruhe und Vernunft, der Gewalt zu begegnen) ... erreicht haben. Ein A. H. (Adolf Hitler) und Genossen ist unter solchen Umständen nicht durch Gewaltlosigkeit zu erledigen. (Einschub am Briefrand: Glauben Sie mir, wir, die wir seinen Aufstieg erlebten, können uns wohl ein Urteil erlauben.) Ein auf allen Gebieten allgemeiner Boykott gegen jeden Angreifer ist heute nicht zu erreichen. Das ist leider eine Tatsache, 
die wir nicht leugnen können. Es scheint mir, daß die Menschheit erst durch eine harte Schule gehen muß - wie dieser wahnsinnige, ruchlose, teuflische, totale Krieg ist - um endlich zu Vernunft und einer guten Gemeinschaft der Völker zu gelangen. ${ }^{68}$

\section{1945: Die Folgen für Westdeutschland}

Das Ende des Feminismus in Deutschland und seiner internationalen Beziehungen dauerten wesentlich länger als die Zeit nationalsozialistischer Herrschaft. Nicht nur die »Unfähigkeit zu trauern« über eine Vergangenheit, die nicht zu »bewältigen « war, Verdrängen und Vergessen trafen zusammen mit dem Verlust der nationalen Identität und der Teilung Deutschlands zwischen den in der Nachkriegszeit dominierenden Blocksystemen in Ost und West. Obwohl die für die »Überlebensarbeit « unentbehrliche Rolle der Frauen in der ersten Nachkriegszeit immer wieder betont wurde, bedeutete dies doch keinen Machtzuwachs für die Mehrheit der Frauen. In allen vier Besatzungszonen waren gleich 1946/47 die sogenannten Frauen-Ausschüsse gegründet worden, die die Beseitigung der Trümmer und den demokratischen Aufbau von unten tatkräftig in die Hand zu nehmen versuchten. Diese Initiatorinnen der »Ersten Stunde« waren in der Mehrheit Vertreterinnen der alten Frauenbewegung und ihrer Organisationen bzw. Politikerinnen aus der Arbeiterbewegung, die unter Verfolgung und KZ-Haft gelitten hatten. ${ }^{69}$

$\mathrm{Zu}$ der ersten Gruppe der Frauenbewegten gehörte Agnes von Zahn-Harnack, die letzte Vorsitzende des Bundes Deutscher Frauenvereine, die der Gleichschaltung zur NS-Organisation 1933 durch Selbstauflösung des Bundes klug zuvorgekommen war. Politisch integer und widerständig gegenüber dem Nationalsozialismus war sie als eine der ersten nach 1945 von den vier Besatzungsmächten Berlins ausgewählt worden, um die deutschen Frauen, ja, die Frauenbewegung 1946 anläßlich einer Einladung nach England im Ausland zu vertreten. ${ }^{70}$ Andere Weggefährtinnen wie Dorothee von Velsen, Marie-Elisabeth Lüders, auch Gertrud Bäumer versuchten ebenfalls, an ihre gemeinsame Vorgeschichte, die politische Tradition und die persönlichen Verbindungen aus der Vorkriegs-Frauenbewegung anzuknüpfen. Einige der alten Mitstreiterinnen waren auf die östliche Seite geraten und sind damit bis jetzt der westlichen Geschichtsschreibung nahezu entgangen: z.B. Else Lüders, die Weggefährtin Minna Cauers, die von Ostberlin aus im Demokratischen Frauenbund Deutschlands (DFD), seit 1947 Dachorganisation der ostdeutschen Frauenverbände, zur Ehrenpräsidentin aufgerückt war. ${ }^{71}$ In dieser Nachkriegszeit wurde, zumindest öffentlich, nicht danach gefragt, was aus den früheren Weggefährtinnen, den Emigrantinnen, insbesondere den Jüdinnen, Linken, Pazifistinnen und Radikalen, geworden war, von denen keine sofort nach 1945 nach Westdeutschland zurïckkehrte.

Der Weg von den ersten Fraueninitiativen bis zu einer neuen westdeutschen Frauenorganisation und ihrer Vertretung auf der internationalen Ebene war noch weit. Dabei war die Friedenssehnsucht aller Menschen wohl niemals größer, waren die Frauen nach dem Zweiten Weltkrieg bereit und überzeugt davon, ihren Teil zu einer besseren, friedlichen Welt beitragen zu müssen. Zahlreiche Friedens- 
initiativen und Friedenskongresse in Ost und West, die schnelle Reorganisation der Internationalen Frauenliga für Frieden und Freiheit oder die Weltbewegung der Mütter, die 1947 eine "Charta der Mütter« auf den Weg brachte, schließlich die von Dorothy Thompson angestoßene Weltorganisation der Mütter aller Nationen (W.O.M.A.N.) scheinen zu belegen, welche Öffentlichkeit ein speziell »weiblicher Pazifismus « in dieser Zeit hatte. Und doch gewinnt die radikale pazifistische Tradition der Frauenbewegung keine Geltung und keine Basis, im Gegenteil. Die Pazifistinnen, insbesondere auch aus der IFFF, werden sehr bald ausgebootet, der Kollaboration mit dem Kommunismus verdächtigt und in der Frontstellung des Kalten Krieges aufgerieben.

Der Versuch, mit der nationalsozialistischen Vergangenheit zu leben und sie zu bearbeiten, wurde sehr unterschiedlich gehandhabt. In den ersten Presseveröffentlichungen ist die Thematik noch präsent, die Notwendigkeit zu politischer Einmischung gerade auch von Frauen wird mit ihrer zweideutigen Rolle in der NS-Vergangenheit begründet, und es wird versucht, die Politikverdrossenheit der sogenannten skeptischen Generation zu überwinden. In dem zunehmenden Schweigen und Verdrängen drückt sich auch ein Generationenwechsel aus. Eine neue Garde von Frauen, die jünger und von der Vergangenheit anscheinend unbelastet sind, benutzen die Förderung zu frauenpolitischem Engagement durch die Besatzungsmächte für eine politische Karriere "Fern von jedem Suffragettentum «. ${ }^{72}$

Der schließlich 1949 aus dem Zusammenschluß der westdeutschen staatsbürgerlichen Frauenverbände gegründete Deutsche Frauenring (DFR) verstand sich als Nachfolgeorganisation des Bundes Deutscher Frauenvereine (BDF), und doch ist diese Kennzeichnung unangemessen. Denn er war eine verhältnismäßig kleine Gruppierung von Funktionärinnen, die die Aufgabe staatsbürgerlicher Bildung mit einer Lobbypolitik im vorparlamentarischen Raum verband. Doch dies genügte, um wenigstens international die westdeutschen Frauenorganisationen zu vertreten und 1951 z.B. in den International Council of Women (ICW) aufgenommen zu werden. $\mathrm{Zu}$ welcher Bedeutungslosigkeit die deutschen Frauenorganisationen im internationalen Zusammenhang inzwischen abgesunken waren, wurde den Beteiligten selbst schmerzlich bewuBt, als im Jahr 1954 ohne große Vordiskussion in einem Entwurf zur Satzungsänderung stillschweigend die bisherigen drei offiziellen Sprachen für Verhandlungen und Schriftwechsel, Englisch, Französisch und Deutsch, auf nur noch zwei offizielle Sprachen reduziert werden sollten.

Auf der Generalversammlung des ICW in Helsinki 1954 versuchte die Vorsitzende des Deutschen Frauenrings (DFR) Else Ulich-Beil vergeblich, die in der Abschaffung von Deutsch als Kongreßsprache liegende symbolische Abwertung und Demütigung rückgängig zu machen. In aller Freundlichkeit und Bestimmtheit gab ihr die schweizerische Präsidentin des ICW zu verstehen:

Realisieren Sie doch, daß es eben viele Menschen auf der Welt und im ICW gibt, die Deutsch nicht mehr hören können und wollen! ${ }^{73}$ 


\section{Anmerkungen}

Dieser Aufsatz beruht auf einem in der Zeitschrift »Ariadne« (24/1993) erschienenen Artikel, der für die "Feministischen Studien* gründlich überarbeitet und erweitert wurde.

1 Ein gutes Beispiel ist der überaus umfangreiche Nachlaß von Rosika Schwimmer, der ungarischen Frauenrechtlerin und Pazifistin, die seit 1921 in Amerika lebte und von hier aus eine weltweite Korrespondenz mit vielen Vertreterinnen der Frauenbewegungen führte (vgl. Rose Rauther, R.S., in: Feministische Studien 1/1984, S. 63-75). Die Schwimmer-Lloyd Collection in der New York Public Library ist mit ihren 1900 Boxen eine unschätzbare Fundgrube, zugleich Zeugnis eines zusammen mit der Historikerin Mary Beard geplanten Weltfrauenarchivs, aber kaum im » Vorbeifahren « zu bearbeiten. Für wichtige Hinweise und freundliches Entgegenkommen während meines viel zu kurzen Besuches möchte ich an dieser Stelle der engagierten Sachwalterin Edith Wynner meinen Dank sagen.

2 Vgl. z. B. Adele Schreiber; Margaret Mathieson, Joumey Towards Freedom, Written for the Golden Jubilee of the International Alliance of Women, Copenhagen 1955; International Council of Women (Hrsg.), Women in a Changing World, The dynamic story of the International Council of Women since 1888, London 1966; M.L. Degen, The History of the Women's Peace Party, New York 1939; Gertrude Bussey; Margaret Tims, Pioneers for Peace: the Women's International League for Peace and Freedom 1915-1965, London 1980; Catherine Foster, Women for all Seasons. The story of the Women's International Leaque for Peace and Freedom, Athen, London 1989.

3 Irmgard Remme, Die internationalen Beziehungen der deutschen Frauenbewegung vom Ausgang des 19. Jahrhunderts bis 1933, Diss. Berlin 1955.

$4 \mathrm{Vgl}$. Gisela Brinker-Gabler (Hrsg.), Frauen gegen den Krieg, Frankfurt a.M. 1980; Sabine Hering; Cornelia Wenzel (Hrsg.), Frauen riefen, aber man hörte sie nicht, 2 Bde., Kassel 1986.

5 Z. B. Marie Stritt (Hrsg.), Der Internationale Frauenkongreß in Berlin 1904. Berlin 1905; Internationales Frauenkomitee für Dauernden Frieden (Hrsg.), Internationaler Frauenkongreß, Haag vom 21. April-1. Mai 1915, Amsterdam 1915.

$6 \mathrm{Im}$ Helene-Lange-Archiv in Berlin, Abt. 17, liegen unter dem Stichwort "Internationaler Frauenbund «, eine Reihe von Materialien und Korrespondenzen aus dem Vorstand des BDF, die jedoch unvollständig und mühsam zu erschließen sind, da oft die andere Seite, die Antwort auf die vorhandenen Briefe, fehlt. Für die sehr hilfreiche Unterstützung bei der Suche nach den einschlägigen Quellen möchte ich deshalb an dieser Stelle Frau Heinrich im Landesarchiv Berlin herzlich danken.

7 Satzung des ICW, zit. n. Jahresbericht der 7. Geschäftsperiode 1920-1922 (HLA Int 2001/11).

8 International Council of Women, Highlights of the History of the ICW, given by Louise C. V. van Eeghen, (Broschüre) Helsinki 1954, S. 3.

9 Einberufen zur Gründungskonferenz des ICW hatte die National Woman Suffrage Association unter der Führung von Elisabeth C. Stanton und Susan B. Anthony, die sich erst 1890 mit der von dem Ehepaar Lucy Stone und Henry Blackwell geführten American Woman Suffrage Association zusammenschloB, vgl. im einzelnen Eleonor Flexner, Hundert Jahre Kampf-Die Geschichte der Frauenrechtsbewegung in den Vereinigten Staaten, Frankfurt/M. 1978.

10 So beklagten sich die Beteiligten des Haager Frauen-Friedenskongresses, daß ihre auf dem Kongre $B$ erarbeiteten Vorschläge für eine neue Friedensordnung zwar im 14-Punkte-Programm des US-amerikanischen Präsidenten Wilson aufgegriffen, aber niemals als solche gewürdigt worden seien. Vgl. Internationale Frauenliga für Frieden und Freiheit; Deutscher Zweig (Hrsg.), Völkerversöhnende Frauenarbeit während des Weltkrieges, München 1920, S. 7.

11 Vgl. Ute Gerhard u.a., Frauenfreundschaften - ihre Bedeutung für Politik und Kultur der alten Frauenbewegung, in: Feministische Studien 1/1993, S. 34.

12 Ich stütze mich hierbei insbesondere auf die im Helene-Lange-Archiv unter der Abt. 17 gesammelte Korrespondenz zum "Internationalen Frauenbund « sowie auf die in der Schwimmer-LloydCollection, New York Public Library, aufgestöberten Briefe deutscher Absenderinnen.

13 "In Deutschland müssen wir mit großem Taktgefühl und konservativen Methoden vorgehen ... Der Unterschied zwischen unserer Situation und der unserer amerikanischen Schwestern ist der 
Tatsache geschuldet, daB sie in einer Republik, wir dagegen in einer Monarchie leben.* Report of the ICW, Washington 1888, S. 219, zit. n. I. Remme, a. a. O., S. 17.

14 Helene Lange, National oder International, abgedr. in: Dies., Kampfzeiten, 1. Bd., Berlin 1928, S. 271.

15 So der vielzitierte Titel von Helmuth Plessner, Die verspätete Nation, in: Günter Dux u. a. (Hrsg.), Gesammelte Schriften, Bd. 6, Frankfurt/M. 1982.

16 Kritisch hierzu David Blackboum; Geoff Eley, Mythen deutscher Geschichtsschreibung. Die gescheiterte bürgerliche Revolution von 1848, Frankfurt/Berlin/Wien 1980.

17 Interessenverbände, die hierbei die Richtung angaben, waren z. B. der Deutsche Kriegerbund (1873), die Deutsche Antisemitische Vereinigung (1886) oder der Verein Deutscher Studenten (1881), der einen AusschluB von Juden in die Satzung aufnahm. Vgl. hierzu ausführlich Otto Dann, Nation und Nationalismus in Deutschland, 1770-1990, München 1993, S. $185 \mathrm{ff}$. Dann berücksichtigt auch die Frauenbewegung als demokratische Bewegung und weist auf das fehlende Frauenwahlrecht als Strukturfehler hin, S. 164.

$18 \mathrm{Vgl}$. stellvertretend Agnes von Zahn-Harnack, Die Frauenbewegung. Geschichte, Probleme, Ziele, Berlin 1928, S. 286.

19 Hierzu Ute Gerhard, Gleichheit ohne Angleichung, München 1990, S.89f.

20 Lange, a. a. O., S. 267.

21 Marie Stritt, Der Anteil der deutschen Frauen an der internationalen Friedensbewegung, in: Handbuch der Frauenbewegung, hrsg. v. H. Lange/G. Bäumer, II. Teil, S. 201.

22 Clara Zetkin, »Reinliche Scheidung «, in: Die Gleichheit Nr. 8/1894.

$23 \mathrm{Vgl}$. die »Resolution, das Frauenwahlrecht betreffend» von 1907, in der Dokumentation in diesem Heft.

24 Brief von Anna Simson an Miss Wilson v. 4. Febr. 1899, HLA Abt. 17, VI, 83 - 328, 4.

25 Auguste Schmidt, Notiz `Zum International Council vom 8. 6. 1899, HLA Abt. 17 IV, 83-328, 5.

26 "Tue andern, wie du willst, daß sie dir tun.»

27 Satzung, zit. n. Geschäftsbericht 1920, vgl. Anm. 7.

$28 \mathrm{Vgl}$. hierzu im Überblick Regine Deutsch, The International Women Suffrage Alliance, London 1929; Schreiber; Mathieson, a. a.O.

29 Schreiber, Mathieson a. a. O., S. 13.

$30 \mathrm{Vgl}$. Remme, a. a. O. , S. 32 u. 34.

31 »Frauen darin zu üben, ihre Bemühungen auf ein gemeinsames Ziel zu konzentrieren. « Brief von M.W. Sewall an M. Stritt vom 19. Okt. 1903, HLA Abt. 17, VI, 83-329, 3.

32 "Obgleich nahezu Fremde, sind wir doch Freundinnen. « Brief von M.W. Sewall an M. Stritt vom 22. Jan. 1901, HLA Abt. 17, VI, 83-329, 3.

33 Brief Stritt an Sewall (ohne Datum, 1899), HLA Abt. 17, IV, 83-329, 1.

34 Stritt an Madam Avril (ohne Datum, 1907/8) HLA Abt. 17, IV, 84-330, 2.

35 »Ich glaube ganz gewiß an internationale Freundschaft ... Wir müssen lernen, unser Gefühl für Menschlichkeit oberhalb des Nationalgefühls anzusiedeln; wir müssen die Nation deshalb nicht weniger lieben, doch der Humanität einen höheren Stellenwert einräumen.« Brief von M.W. Sewall an Anna Simson vom 3. Juni 1899, HLA Abt. 17, IV 83-328, 5.

$36 \mathrm{Vgl}$. hierzu Mineke Bosch with Annemarie Klostermann (Eds.), Politics and Friendship. Letters from the International Woman Suffrage Alliance, 1902-1942, Ohio 1990; Gerhard u. a., Frauenfreundschaften, a. a. O., S. $21 \mathrm{ff}$.

37 Helene Lange, Lebenserinnerungen, Berlin 1925, S. 234.

38 Lange, National oder International, a. a.O., S. 270.

39 Gertrud Bäumer, Die Frau in Volkswirtschaft und Staatsleben, Stuttgart/Berlin 1914, S. 255.

40 Ausführlicher Ute Gerhard, Unerhört. Die Geschichte der deutschen Frauenbewegung, Reinbek 1990, S. $292 \mathrm{ff}$.

41 Gertrud Bäumer, Lebensweg durch eine Zeitenwende, Tübingen 1933, S. 248.

42 Zit. n. Schreiber; Mathieson, a. a. O., S. 25.

43 Zit. n. Deutsch, a. a. O., S. 21: »Es liegt an uns zu beweisen, daß das, was uns verbindet, stärker ist als das, was uns trennt.k

44 Gerhard, Unerhört, a. a.O., S. $308 \mathrm{ff}$. 
45 Vgl. Völkerversöhnende Frauenarbeit 1920, a. a.O., S. $5 \mathrm{ff}$.

46 Brief von Anna Edinger an May W. Sewall, Dez. 1912, HLA Abt. 17, IV, 84-330, 7.

47 Brief von Isabel Aberdeen an Gertud Bäumer vom April 1915, HLA Abt. 17, IV, 84-330, 8.

48 Lida Gustava Heymann; Anita Augspurg, Erlebtes - Erschautes. Deutsche Frauen kämpfen für Freiheit, Recht und Frieden, Meisenheim 1972, S. 121.

$49 \mathrm{Vgl}$. hierzu den Brief von Anna Edinger an Helene Lange in der Dokumentation in diesem Heft.

$50 \mathrm{Vgl}$. Schreiber; Mathieson, a. a. O., S. 29.

51 Else Ulich-Beil, Ich ging meinen Weg, Lebenserinnerungen, Berlin 1961, S. 103.

52 Brief von May W. Sewall an Elisabeth Altmann-Gottheiner vom 29. April 1919, HLA 17. Abt., VI, 84-330, 8; vgl. auch zum folgenden Remme, a.a.O., S. $100 \mathrm{f}$.

53 Vgl. Dora Peyser, Alice Salomon. Die Begründerin des sozialen Frauenberufs in Deutschland, Köln/Berlin 1958, S. 85; Alice Salomon, Charakter ist Schicksal, Lebenserinnerungen, Weinheim/Basel 1983, S. $184 \mathrm{f}$.

54 Brief vom 24. 8. 1920; zit. n. Remme, a. a. O., S. 102.

55 Emma Ender, Erster Bericht über die Generalversammlung des ICW in Washington 1925, zit. $\mathrm{n}$. Remme, a.a.O., S. 111.

$56 \mathrm{Vgl}$. die gleichnamigen Dokumentationsbände hrsg. v. der Internationalen Frauenliga für Frieden und Freiheit, Deutscher Zweig, München 1920 und ff.

$57 \mathrm{Vgl}$. Catherine Forster, a. a.O., S. 18.

58 Brief vom 23. 10. 1927, Schwimmer-Lloyd Coll. Serie A, Box 174.

59 Lida Gustava Heymann, S.O.S., zit. in: Gerhard, Unerhört, S. 358/359.

60 Remme, a. a.O., S. 111.

61 Dorothee von Velsen, Im Alter die Fülle, Erinnerungen, Tübingen 1956, S. 309.

62 Highlights in the History, a. a. O., S. 7; Ulich-Beil, a. a. O., S. 239.

63 Dies zeigten meine Recherchen in der Sophia Smith Collection, Northampton Mass., Box: Peace Organizations, sowie in dem Nachlaß Rosika Schwimmer in der New York Public Library, Schwimmer-Lloyd Collection.

64 Z.B. die Briefe von Helene Stöcker oder von Käthe Braun-Prager, Schriftstellerin und Nachlaßverwalterin von Rosa Mayreder, Schwimmer-Lloyd Coll. Serie G, Box 27.

65 »... als eine der Gründerinnen der IFFF, die keine Gelegenheit versäumte, besonders die jüngeren Frauen daran zu erinnem, daß Frieden ohne Freiheit eine Schande ist, und daß ein gerechter und permanenter Friede nur auf der Basis erworbener und gesicherter Freiheit erreicht werden kann. « Dok. aus der S. Smith Coll. Peace Organizations, Box 6.

$66 \mathrm{Vgl}$. Heymann; Augspurg, Erlebtes - Erschautes ..., a. a. O.

67 Brief v. 4. 2. 1938, Schwimmer-Lloyd Coll. Serie A, Box 300.

68 Brief v. 19. 5. 1942, Schwimmer-Lloyd Coll., Serie A, Box 381.

69 Hierzu und zum folgenden vgl. Ulla Wischermann; Elke Schüller; Ute Gerhard (Hrsg.), Staatsbürgerinnen zwischen Partei und Bewegung. Frauenpolitik in Hessen 1945-1955, Frankfurt/M. 1993.

70 Ilse Reicke, Agnes von Zahn-Harnack, Ein Lebensbild, in: A. von Zahn-Harnack, Schriften und Reden, 1914-1950, Tübingen 1964, S. 205 f.

71 Vgl. Der Silberstreifen (1948) H. 1, S. 38; auch zum folgenden Ute Gerhard, Fern von jedem Suffragettentum, in: Wischermann u. a. Hrsg., Staatsbuirgerinnen ..., a. a.O., S. 13 ff.; vgl. auch Sabine Hering, »Eine Filiale der SED? « Anmerkungen zum Nationalismus des Demokratischen Frauenbundes Deutschlands, in: Ariadne Nr. 24, 1993, S. 43-48.

72 Theanolte Bähnisch, in: Die Stimme der Frau (1948), H. 1, S. 25, zit. n. Annette Kuhn, Hrsg., Frauen der deutschen Nachkriegszeit, Düsseldorf 1986, Bd. 2, S. 226.

73 Ulich-Beil, a.a.O., S. 243. 